\title{
Exploiting Chemical Diversity through C-H Activation and DNA-Encoding
}

Zhoulong Fan, Shuai Zhao, Tao Liu, Peng-Xiang Shen, Zi-Ning Cui, Zhe Zhuang, Qian Shao, Jason Chen, Anokha S. Ratnayake, Mark E. Flanagan, Dominik K. Kölmel, David W. Piotrowski, Paul Richardson, jin-quan yu

Submitted date: 05/01/2020 - Posted date: 08/01/2020

Licence: CC BY-NC-ND 4.0

Citation information: Fan, Zhoulong; Zhao, Shuai; Liu, Tao; Shen, Peng-Xiang; Cui, Zi-Ning; Zhuang, Zhe; et al. (2020): Exploiting Chemical Diversity through C-H Activation and DNA-Encoding. ChemRxiv. Preprint. https://doi.org/10.26434/chemrxiv.11514774.v1

DNA-encoded library (DEL) technology has the potential to dramatically expedite hit identification in drug discovery owing to its ability to perform protein affinity selection with millions or billions of molecules in a single experiment. To expand the molecular diversity of DEL, it is critical to develop different types of DNA-encoded transformations that produces billions of molecules with distinct molecular scaffolds. Sequential functionalization of multiple $\mathrm{C}-\mathrm{H}$ bonds provides a unique avenue for creating diversity and complexity from simple starting materials. However, the use of water as solvent, the presence of DNA, and the extremely low concentration of DNA-encoded coupling partners $(0.001 \mathrm{M})$ have hampered the development DNA-encoded $\mathrm{C}\left(\mathrm{sp}^{3}\right)-\mathrm{H}$ activation reactions. Herein, we report the realization of palladium-catalyzed $\mathrm{C}\left(\mathrm{sp}^{3}\right)-\mathrm{H}$ arylation of aliphatic carboxylic acids, amides and ketones with DNA-encoded aryl iodides in water. Notably, the present method enables the use of alternative sets of bifunctional building blocks, and facilitates access to certain setups for DELs. Furthermore, sequential C-H arylation chemistry enabled the on-DNA synthesis of structurally-diverse scaffolds containing enriched $\mathrm{C}\left(\mathrm{sp}^{3}\right)$ character, chiral centers, cyclopropane, cyclobutane, and heterocycles.

File list (2) 
Other files

SICHActivationDELYuChemRxiv.pdf (14.48 MiB)

CHActivationDELYuChemRxiv.pdf (1.67 MiB) view on ChemRxiv - download file view on ChemRxiv - download file 